\title{
Necessidades, expectativas, potencialidades e fragilidades dos espaços de lazer nas comunidades quilombolas em Garanhuns-PE
}

Needs, expectations, potentialities and weaknesses of leisure spaces in quilombola communities in Garanhuns-PE

Necesidades, expectativas, potencialidades y debilidades de los espacios de ocio en las comunidades quilombola de Garanhuns-PE

Maria Deisyelle Sibaldina da Silva Almeida ORCID: https://orcid.org/0000-0001-8997-0334 Universidade de Pernambuco, Brasil E-mail: mariadeisyelle@gmail.com Nicole Caroline Nascimento da Silva ORCID: https://orcid.org/0000-0001-6425-8324 Universidade de Pernambuco, Brasil E-mail: nicole-carolinee@hotmail.com

Taynara Poliana Gonçalves de Melo ORCID: https://orcid.org/0000-0001-6802-0313 Universidade de Pernambuco, Brasil

E-mail: taynaragoncalvesdemelo@gmail.com

Fernando de Lima

ORCID: https://orcid.org/0000-0001-8060-8523 Universidade de Pernambuco, Brasil E-mail: fernandolima.ef@gmail.com

Itamar Lages

ORCID: https://orcid.org/0000-0001-6698-0150 Universidade de Pernambuco, Brasil E-mail: itamar.lages@upe.br

Ranieri Moreira da Silva ORCID: https://orcid.org/0000-0002-2190-9796 Universidade de Pernambuco, Brasil E-mail: moreira201534@gmail.com

Wellington da Silva Rodrigues Junior ORCID: https://orcid.org/0000-0002-5314-5890 Universidade de Pernambuco, Brasil E-mail: wellington.junior@upe.br

Emmanuelly Correia de Lemos ORCID: https://orcid.org/0000-0003-1450-6160 Secretaria Estadual de Saúde de Pernambuco, Brasil E-mail: emmanuellylemos@gmail.com

\section{Resumo}

O estudo teve como objetivo identificar as necessidades, expectativas, potencialidades e fragilidades quanto aos espaços de lazer na comunidade quilombola Estivas-Garanhuns/PE. A pesquisa caracterizou-se como exploratória e descritiva com abordagem qualitativa. Participaram da pesquisa 30 moradores. As entrevistas foram gravadas e posteriormente transcritas e os dados foram analisados por meio de análise de conteúdo. Após análise emergiram duas categorias, sendo elas: necessidades e expectativas; potencialidades e fragilidades da comunidade quilombola quanto ao espaço de lazer. As necessidades e expectativas quanto a espaços de lazer surgiu como sendo um dispositivo que proporciona saúde. As potencialidades indicadas foram a articulação das lideranças para construção do espaço com os órgãos governamentais e lideranças religiosas, bem como, a existência de equipamentos sociais do território e materiais recicláveis na comunidade. As fragilidades foram a falta de incentivo financeiro por parte dos órgãos públicos e a falta de uma maior mobilização da comunidade para construção do espaço de lazer. Através da roda de conversa com os participantes foi proposto coletivamente elementos que contribuíssem para a construção do plano de ação e desenvolvimento do espaço de lazer que contribuirá para promoção da saúde dos moradores de forma sustentável.

Palavras-chave: Sistema Único de Saúde; Promoção da saúde; Saúde da população rural; Meio ambiente e saúde pública; Centros de convivência e lazer.

\section{Abstract}

The study aimed to identify the needs, expectations, strengths and weaknesses regarding leisure spaces in the Quilombola community Estivas-Garanhuns/PE. The research was characterized as exploratory and descriptive with a 
qualitative approach. Thirty residents participated in the survey. The interviews were recorded and later transcribed and the data were analyzed using content analysis. After analysis, two categories emerged, namely: needs and expectations; strengths and weaknesses of the quilombola community in terms of leisure space. The needs and expectations regarding leisure spaces emerged as a device that provides health. The indicated potentialities were the articulation of the leaders for the construction of the space with government bodies and religious leaders, as well as the existence of social equipment in the territory and recyclable materials in the community. The weaknesses were the lack of financial incentive from public agencies and the lack of greater mobilization of the community to build the leisure space. Through the conversation circle with the participants, elements were collectively proposed that would contribute to the construction of the action plan and development of the leisure space that will contribute to promoting the health of residents in a sustainable way.

Keywords: Unified Health System; Health promotion; Rural population health; Environment and public health; Community and leisure centers.

\section{Resumen}

El estudio tuvo como objetivo identificar las necesidades, expectativas, fortalezas y debilidades de los espacios de ocio en la comunidad quilombola Estivas-Garanhuns / PE. La investigación se caracterizó por ser exploratoria y descriptiva con enfoque cualitativo. Treinta residentes participaron en la encuesta. Las entrevistas fueron grabadas y posteriormente transcritas y los datos fueron analizados mediante análisis de contenido. Después del análisis, surgieron dos categorías, a saber: necesidades y expectativas; fortalezas y debilidades de la comunidad quilombola en términos de espacio de ocio. Las necesidades y expectativas con respecto a los espacios de ocio surgieron como un dispositivo que brinda salud. Las potencialidades señaladas fueron la articulación de líderes para construir el espacio con agencias gubernamentales y líderes religiosos, así como la existencia de equipamiento social en el territorio y materiales reciclables en la comunidad. Las debilidades fueron la falta de incentivo financiero de las agencias públicas y la falta de una mayor movilización de la comunidad para construir el espacio de esparcimiento. A través del círculo de conversación con los participantes, se propusieron colectivamente elementos que contribuirían a la construcción del plan de acción y desarrollo del espacio de ocio que contribuirá a promover la salud de los residentes de forma sostenible.

Palabras clave: Sistema Único de Salud; Promoción de la salud; Salud de la población rural; Medio Ambiente y Salud Pública; Centros comunitarios y de ocio.

\section{Introdução}

O espaço rural brasileiro é vivo e dinâmico, sendo habitado por diversas populações que possuem culturas distintas. Nesse espaço, encontra-se uma diversidade de povos, sejam eles camponeses da agricultura familiar, trabalhadores rurais acampados e/ou assentados, povos tradicionais como as comunidades quilombolas, ribeirinhas, povos indígenas e povos que habitam ou usam reservas extrativistas em áreas florestais ou aquáticas, populações atingidas por barragens, entre outras (Pnsipcf, 2013).

Essas populações ainda são historicamente oprimidas, tendo a negação de direitos básico como o acesso à educação de qualidade, a saúde, ao lazer, ao trabalho digno, a terra para viver e plantar alimentos saudáveis, a liberdade de existir com suas identidades, e a vida plena na sua integralidade. Tais populações vem resistindo e lutando incessantemente diante de um genocídio sistemático e estruturado para com os povos do campo, florestas e das águas. Essas populações assumiram o nome de Campo ao invés de rural, justamente devido a realidade citada (Dias, 2011).

Pernambuco, estado do Nordeste brasileiro, foi também marcado pela luta dos povos negros contra escravidão, sendo os quilombos os maiores pontos de resistência a esse processo. Foi palco de muitos conflitos no contexto rural, como as Ligas Camponesas nas lutas pela terra e a formação de muitos quilombos, compostos por africanos escravizados que fugiram principalmente do massacre do Quilombo de Palmares (Ferreira, 2011). O município de Garanhuns é uma das regiões que pode ser vista como marco dessa história de resistência vivida pelo povo negro quilombola em Pernambuco. A cidade está situada no Agreste, localizada a $230 \mathrm{~km}$ da capital, Recife, sendo território marcado por conflitos intensos entre quilombolas e proprietários de terra e imobiliárias que se apropriaram indevidamente dos territórios de resistência (dos quilombos).

No período em que no Brasil vigorava a lei das Capitanias hereditárias, Pernambuco foi dividido em diversas Sesmarias (lote de terras distribuído a um beneficiário, com o objetivo de cultivar nas terras), a Sesmaria dos Aranhas, é onde se localizava a área da atual cidade de Garanhuns-PE. Nesse período, foram construídas diversas comunidades quilombolas como: Estivas, 
Castainho, Tigre, Timbó, Caluete e Estrela, formadas principalmente por quilombolas remanescentes do quilombo de Palmares. Porém, nem todas as comunidades possuem a nomeação de terras quilombolas. São populações que lutam para sair da marginalidade e fazer valer os direitos conquistados nas últimas décadas (Diagnóstico de saúde, 2019).

Estivas, a comunidade a ser estudada, compreende a aproximadamente $1 \mathrm{~km}^{2}$ e nesse perímetro residem 168 famílias, totalizando 366 pessoas (Diagnóstico de saúde, 2019). Estivas possui a nomeação de terra quilombola, ação importante de reconhecimento dos direitos quilombolas, intensificada com o decreto $n^{\circ} .4 .887 / 2003$ que regulamenta o procedimento para identificação, reconhecimento, delimitação, demarcação e titulação das terras ocupadas por remanescentes das comunidades dos quilombos, de acordo com a Constituição Federal, de 1988, em Art. 68, do Ato das Disposições Constitucionais Transitórias (ADCT).

Durante a construção do diagnóstico de saúde realizado pela equipe da Residência Multiprofissional em Saúde da Família com Ênfase na População do Campo (RMSFC), pôde-se verificar a não existência de espaços públicos de lazer, para o descanso, o exercício físico e a recreação infantil na comunidade. Com isso há uma relação de aumento da probabilidade de problemas de saúde, já que, a saúde é multifatorial, devendo-se considerar os aspectos físicos, mental e social e não apenas a ausência de doenças, bem como, de acordo com a lei 8080/90 art. $3^{\circ}$. a saúde tem como fatores determinantes e condicionantes, entre outros, a alimentação, a moradia, a educação, o lazer e o acesso aos bens e serviços essenciais, entre outros (Gerais,1990).

O lazer faz parte das necessidades fundamentais do indivíduo para obter melhor qualidade de vida. Historicamente o lazer foi compreendido como uma fração de tempo situado no âmbito do chamado "tempo livre", tempo esse conquistado por trabalhadores nas lutas por diminuição de jornada de trabalho e pelo gozo de fins de semana e férias remuneradas (Freitas, 2009). Os modos de lazer dos quilombolas estão relacionados ao que a igreja e seus cultos oferecem como as festas em louvor aos santos da Igreja Católica e aos guardamentos (velórios), a prática do futebol é presente na comunidade, porém, somente o público masculino a realiza, cabendo às mulheres observar essa prática aos redores do campo, realizar serviços domésticos e assistirem as novenas (Rangel, Mirand \& Lara, 2014).

O lazer e o esporte caracterizam-se como o espaço e tempo de uma necessária reconstrução cultural, essenciais para a realização plena da condição humana podendo exercer um significativo papel social, político, econômico e cultural, se forem assumidos enquanto cultura vivenciada no tempo disponível, e como um direito inalienável de todo cidadão. Possui ainda uma importância especial, por caracterizar-se como espaço de encontro e de convívio. O lazer deve ser entendido mais além, constituindo-se em uma experiência de desenvolvimento ocorrida dentro do tempo-espaço disponível (Viana et al., 2017).

O lazer dos quilombolas em relação ao espaço urbano acaba comprometido dada a escassez, pelos órgãos públicos, de oferta de oportunidades que possam intensificar a relação cultural entre os moradores do quilombo e os citadinos.

Diante disso, este estudo teve como objetivo identificar as necessidades, expectativas, potencialidades e fragilidades quanto a espaço de lazer na comunidade quilombola Estivas - Garanhuns/PE. Como também, elencar proposta para a construção conjunta com a comunidade do plano de ação e desenvolvimento do espaço de lazer comunitário.

\section{Metodologia}

O estudo caracterizou-se como exploratório e descritivo de abordagem qualitativa. Foram convidados 30 moradores da comunidade Quilombola Estivas do município de Garanhuns do Agreste do estado de Pernambuco, no período de julho a dezembro de 2020. Desenvolver este estudo em meio a pandemia da Covid-19 foi um desafio e limitou a realização da pesquisa de acordo com o cronograma previsto, bem como a participação de mais moradores. Os critérios de inclusão para participar da pesquisa foram: ser adolescente e obter a autorização dos pais, adulto ou idoso, ser morador há mais de cinco anos na comunidade e que aceitasse participar da pesquisa por meio da assinatura do Termo de Consentimento Livre e Esclarecido (TCLE). 
A coleta de dados foi realizada em duas etapas: a primeira conduzida por meio de entrevistas, realizadas no território respeitando o distanciamento social, uso das máscaras e condutas de higiene. O roteiro da entrevista foi composto por perguntas que buscou identificar às necessidades e expectativas dos moradores da comunidade quilombolas e as potencialidades e fragilidades locais do quilombo de Estivas Garanhuns-PE, quanto o entendimento do conceito de espaços de lazer, sua relação com a saúde e caminhos para construção desse espaço na comunidade.

Os dados foram analisados por meio de análise de conteúdo, seguindo o método de Bardin (2011). Após análise dos resultados das entrevistas, partiu-se para segunda etapa por meia da roda de conversa, em que foram convidados 10 entrevistados, desses 8 compareceram a roda de conversa para elencar propostas para a construção do plano de ação quanto ao espaço de lazer na comunidade. A roda foi realizada na Unidade de Saúde da Família Estivas, a partir da seguinte metodologia: acolhimento, apresentação dos objetivos da roda de conversa, apresentação dos resultados da primeira etapa, discussão e encaminhamentos quanto à construção conjunta com a comunidade do plano de ação para a construção e desenvolvimento do espaço de lazer.

A pesquisa seguiu as diretrizes éticas da Resolução n 466/2012 e foi consentida pela Secretaria de Saúde do município de Garanhuns com assinatura da carta de anuência, sendo aprovada no Comitê de Ética em Pesquisa. Todos os participantes assinaram o TCLE.

\section{Resultados e Discussão}

A população do estudo foi constituída por 30 moradores da comunidade quilombola de Estivas, sendo 80,0\% do sexo feminino, $66,7 \%$ adultos, $20,0 \%$ idosos e $13,3 \%$ de adolescentes. No quesito raça/cor $53,0 \%$ se declaram preto e $37,0 \%$ pardos. Os resultados encontrados nas entrevistas foram descritos considerando as categorias de análise quanto a espaço de lazer na comunidade em estudo: 1. Necessidades e expectativas; 2. Potencialidades e fragilidades, e suas subcategorias.

É importante refletir acerca da compreensão da saúde enquanto multifatorial considerando todos os aspectos para obter uma melhora na qualidade de vida em sua integralidade, pautando-se no fortalecimento de políticas de promoção à saúde junto às necessidades do território numa perspectiva popular. O lazer também está compreendido como um direito dentro das políticas de promoção da saúde como o incremento da consciência política da população para sua organização sobre as necessidades de ambientes favoráveis à saúde.

Diante disso, as necessidades e expectativas quanto a espaços de lazer na comunidade quilombola se expressaram nas seguintes categorias de análises:

\section{Compreensão de saúde e sua promoção}

Com relação a compreensão de saúde e sua promoção, os entrevistados consideraram que saúde está relacionada a um conceito complexo, e sua promoção se dá através de bem-estar físico e mental, a família, hábitos saudáveis, e viver em harmonia. Conforme pode ser verificado nas falas a seguir:

\section{[...] Estar bem psicologicamente e mentalmente, e bem consigo mesmo e com o meio que vive [...] (P15).}

[...] Saúde é tudo, sem saúde a gente não vive [...] (P1).

[...] Saúde é tudo, com saúde a gente trabalha, é estar bem [...] (P8).

Ao falarem dessa forma, a saúde deixa de ser compreendida a partir de uma perspectiva médica curativa, e passa a ser concebida como um processo que envolve promoção e prevenção de saúde, fortalecendo a saúde ampliada. Pensar na saúde dessa forma está alinhada ao conceito multifatorial, devendo-se considerar os aspectos físico, mental e social e não apenas a ausência de enfermidades (Scliar, 2007). De acordo com a Organização Mundial da Saúde (OMS), ampliar o sentido dos 
processos de saúde e doença é passar a considerá-los algo mais complexo e diretamente relacionado a fatores políticos, econômicos, ideológicos e de representação social (Ribeiro \& Wilson, 2007).

Muitos moradores relataram que a Unidade de Saúde da Família (USF) é o que promove saúde na comunidade, sendo conhecida como a porta de entrada dos usuários no Sistema Único de Saúde (SUS). Da mesma forma foi relatado como promotora de saúde, o grupo de exercício físico que é proporcionado no espaço da associação dos moradores de Estivas pelos profissionais residentes de Educação Física e do Núcleo Ampliado de Saúde da Família e Atenção Básica (NASF-AB). E os saberes populares que através dos conhecimentos e da utilização das plantas, dos chás que são obtidas principalmente através do cultivo em quintais, sendo elas citadas como promotora de saúde no território:

\section{[...] O posto de saúde e o grupo de exercício físico [...] (P13).}

[...] O posto de saúde, os chás que a gente usa [...] (P4).

[...] O posto de saúde, os chás que têm na natureza, o ar puro da comunidade é o que promove saúde no território [...] (P8).

A saúde é um direito fundamental de qualquer ser humano, sem discriminação de raça, cor, sexo ou condição socioeconômica. Baseando-se neste direito, a Promoção da Saúde oferece um conceito positivo e inclusivo de saúde como um determinante da qualidade de vida, incluindo o bem-estar mental e espiritual (Durand, 2016).

Com a promoção de saúde reforça o entendimento da saúde como um direito de todos e dever do Estado. Com a aprovação da Lei № 8.080 de 1990, passa a ser um componente fundamental das políticas e programas públicos em todos os países na busca de equidade e melhor saúde para todos. Ressaltando a Política Nacional de Promoção da Saúde (PNPS), que tem como objetivo promover a qualidade de vida e reduzir vulnerabilidades e riscos à saúde relacionados aos seus determinantes $\mathrm{e}$ condicionantes, modos de viver, condições de trabalho, habitação, ambiente, educação, lazer, cultura, acesso a bens e serviços essenciais (Malta, 2014).

\section{Compreensão sobre espaços de lazer}

Para os entrevistados, o espaço de lazer é entendido como um espaço de descansar, conversar, brincar e praticar exercício físico. O lazer faz parte das necessidades fundamentais do indivíduo para obter melhor qualidade de vida, independentemente das idades. De acordo com as falas dos entrevistados:

[...]É um lugar como uma praça, para conversar e relaxar [...] (P2).

[...] É um espaço onde as pessoas vão conversar, se divertir, caminhar e se exercitar [...] (P10).

[...] Lugar de encontros, de se exercitar e de brinquedos. Promovendo assim a promoção da saúde [...] (P 28).

Com a presença desses espaços de lazer alcança não somente benefícios biológicos, mas interferem também em outros determinantes sociais que desencadeiam resultados na manutenção da saúde como um todo (Batista, 2012).

Os participantes relatam que na comunidade não tem um espaço destinado para o lazer com adesão para todos. O mesmo, no território é caracterizado por bares, clubes privados, igrejas católicas, evangélicas e campo de futebol que tem a maior adesão dos homens. Porém, esses espaços não são frequentados por todos os moradores e eles sentem falta de espaços que sejam destinados para diversão, como praças e lugares para realizar exercício físico. Diante disso, todos os participantes responderam que têm interesse em ter esse espaço de lazer na comunidade. Isto pode ser visualizado nos trechos das falas de alguns participantes ao serem perguntados se teriam interesse de ter um espaço de lazer na comunidade? 
[...] Sim. Aqui não tem [...] (P 3).

[...] Sinto sim, porque seria para todas as pessoas. Principalmente para as pessoas que têm depressão [...] (P 28).

[...] Sinto $\operatorname{sim}[\ldots]($ P30).

No que se refere ao lazer, verifica-se uma precariedade no acesso aos instrumentos e aos equipamentos, da mesma forma que não há uma clara percepção dessa área como direito e necessidade humana (Freitas, 2009). Para superar essas desigualdades vivenciadas pelas pessoas em desvantagem social e educacional e as mais afastadas, requer-se políticas que procurem ampliar o acesso daquelas pessoas a bens e serviços promotores de saúde e criar ambientes favoráveis, estabelecendo-se alta prioridade aos grupos mais desprivilegiados e vulneráveis, reconhecendo como peculiar a cultura de povos indígenas, quilombolas, minorias éticas e imigrantes (Santos Lacerda, 2018).

Diante disso, o lazer dos quilombolas em relação ao espaço urbano acaba comprometido dada a escassez, pelos órgãos públicos, de oferta de oportunidades que possam intensificar a relação cultural entre os moradores do quilombo e os citadinos. Ressaltando que os espaços de lazer fazem parte das necessidades fundamentais do indivíduo para obter melhor qualidade de vida (Ferreira, 2011).

\section{Espaços de lazer e promoção da saúde}

Os moradores entendem que lazer faz parte das necessidades essenciais do indivíduo e a sua presença tem como resposta positiva o bem-estar físico e mental. Com isso, todos os participantes relataram a importância desses espaços de lazer para a promoção de saúde de acordo com as seguintes falas:

[...] Sim, tanto física como mental [...] (P15).

[...] Sim, porque quando a gente sai distrai a mente e quando fica em casa a gente só pensa em besteira [...] (P3).

[...] Sim, porque a gente faz caminhada, se distrai e conversa [...] (P30).

[...] Sim, porque melhoraria mais a nossa saúde, hoje em dia muita gente morre de infarto, diabético e depressão, se tivesse academia da saúde isso poderia ser diferente [...] (P5).

As respostas afirmam que o lazer resulta em muitos benefícios, isso convém com o estudo de (Freitas, 2009), em que as vivências lúdicas praticadas nos grupos sociais favorecem não somente a manutenção e perpetuação da cultura, mas contribuem como fator de promoção da saúde. Referente às doenças crônicas não transmissíveis citadas nas respostas, por exemplo a hipertensão representa um dos principais problemas de saúde pública nas populações negras em todo o mundo e, no Brasil, por ser mais frequente em populações afrodescendentes, destaca-se no contexto das doenças mais importantes por razões étnicas (Bezerra, 2013). Com isso, a promoção de saúde e a minimização de fatores adversos de doenças crônicas têm que ser levado em consideração as relevâncias da sua efetividade, e isso foi identificado durante a pesquisa.

Em relação às potencialidades e fragilidades da comunidade quilombola quanto aos espaços de lazer, obteve-se as seguintes categorias de análises:

\section{Potencialidades de espaços de lazer na comunidade quilombola}

Todos os participantes afirmaram que a comunidade tem condição de ter esse espaço. Principalmente pelo motivo do território ser amplo, tendo vários lugares a serem pensados. A maior parte dos entrevistados citou o terreno ao lado da Unidade de Saúde da Família Estivas (USF-Estivas) e ao lado da igreja católica de Estivas. 
[...] Sim, porque tem muito espaço [...] (P6).

[...] Sim, porque seria bom para todas as comunidades [...] (P8).

[...] Perto do posto de saúde [...] (P11).

[...] Perto do posto ou perto da escola [...] (P24).

[...] Perto do posto de saúde ou da Igreja [...] (P4).

O espaço ao lado da USF foi proposto por ser um local frequentado por muitas pessoas do território e das comunidades vizinhas, por ser um lugar centralizado e perto da escola de Estivas, assim seria usufruído por todos. Ao lado da USF já existe um espaço grande e plano que antigamente era realizado as festas culturais do território, e atualmente as festividades são realizadas em outro local. Outro lugar citado foi perto da igreja católica da comunidade Estivas, ao lado dela possui um terreno plano, onde já surgiu esse interesse durante uma das reuniões com os grupos religiosos, porém, não se articularam para planejar e executar.

A criação desses ambientes favoráveis à saúde, no campo da saúde, foi refletida e consolidada na Constituição Federal de 1988, quando houve a construção de uma nova política de saúde no Brasil. Política que tenha um olhar integral sobre o ambiente em todas as suas dimensões, onde estavam inseridos os indivíduos e suas famílias, incentivando, desta forma, a criação de estratégias (Cohen et al., 2007).

Todos os participantes responderam que há o desejo de ter esse espaço de lazer na comunidade, e ainda, demonstraram interesse em participar da construção e manutenção desse espaço. Compreendendo o lazer como manifestação importante para a preservação da identidade cultural de um povo é essencial para a promoção e manutenção da saúde e qualidade de vida da coletividade, fazendo necessário a valorização e manutenção desses espaços.

Alguns idosos relataram não ter condições de ajudar devido à idade e algumas limitações do corpo. Porém, a vontade de contribuir é maior que as dificuldades, isso é percebido na fala a seguir;

[...] Sim, se eu não pudesse por causa da minha idade eu carregava ao menos um tijolo para ajudar [...] (P 28).

[...] Sim, mas não posso, pelo fato da idade [...] (P27).

[...] Sim. Muita gente ia [...] (P9).

Esse desejo de ajudar e contribuir pode ser chamado de intersetorialidade que é uma ação de promoção da saúde modificadora dos determinantes sociais de saúde. De acordo com Warschauer (2014) essa perspectiva, de ações Inter setoriais têm se mostrado como uma estratégia importante na busca de novos modelos organizacionais e pode ser entendida como uma articulação de saberes e experiências no planejamento, realização e avaliação de políticas, programas e projetos dirigidos a comunidades, nesse caso a comunidade Quilombola Estivas, com o objetivo de atender às suas necessidades e expectativas de forma participativa e integral do território.

Ao questionar se teria alguém ou algum órgão que poderia ajudar na elaboração do projeto, todos responderam que os políticos do município e a associação de moradores (comunidade) de Estivas, e algumas pessoas mencionaram a igreja, como potencializador na ajuda do planejamento, elaboração e ação para o projeto. De acordo com as falas:

[...] Associação, igreja, prefeitura e a comunidade [...] (P15).

[...] Prefeitos, vereadores e a população da comunidade [...] (P16).

[...] Os governantes e a comunidade [...] (P23). 
A promoção da qualidade de vida não é, portanto, uma tarefa individual e não depende apenas da comunidade, mas se insere como uma responsabilidade sociocultural, envolvendo as autoridades competentes com a responsabilidade de implementar políticas públicas que atendam às necessidades básicas dos sujeitos de direitos (Freitas, 2009).

Os moradores da comunidade são articulados com a associação dos moradores de Estivas, facilitando os projetos e as conquistas de interesse coletivo. E quando questionados se teria alguém ou algum órgão que poderia dificultar a criação desse espaço de lazer? A maioria dos participantes responderam que pode ter pessoas de má índole, poderá ter pessoas que não queiram contribuir e pôr fim a questão financeira que poderá dificultar a criação desse espaço. Esses foram os resultados quanto a dificuldades que poderiam ser encontradas na elaboração desses espaços de lazer.

\section{[...] Pode ter sim, porque tem gente de coração bom e gente de coração ruim [...] (P8).}

[...] A questão financeira para ser executado [...] (P16).

[...] Acho que não, pode ter gente que não queira participar [...] (P26).

Os resultados encontrados nas entrevistas foram apresentados e discutidos na roda de conversa com os participantes. Após o debate sobre as necessidades, expectativas, potencialidades e fragilidades da comunidade quanto ao espaço de lazer, foram definidos alguns componentes para elaboração conjunta do plano de ação de construção e desenvolvimento do espaço de lazer. Esse plano de ação será entregue à associação de moradores de Estivas, a USF Estivas, a nova turma de RMSFC e aos gestores e técnicos do município de Garanhuns-PE, para apoiarem a construção e manutenção do espaço de lazer na comunidade.

Além disso, vale ressaltar que ainda há uma fragilidade de publicação de estudos sobre o tema de lazer no contexto da população do campo, o que pode ter agregado também algumas limitações para construção desse estudo.

\section{Conclusão}

O lazer, assim como a saúde, é um direito social previsto na Constituição Brasileira. No que diz respeito aos aspectos necessidade e expectativa dos espaços de lazer, o estudo identificou que estes espaços são entendidos como um dispositivo que proporciona saúde. Por outro lado, foi identificada a ausência desses espaços de lazer, como: praça (espaços com bancos, mesas), quadra poliesportiva e parques (brinquedos infantis, balanço, gangorra e escorrego).

As articulações das lideranças comunitárias com os órgãos governamentais apresentaram-se como grande potencialidade para construção de espaços de lazer na comunidade quilombola, assim como, o interesse das lideranças religiosas nessa articulação com os moradores da comunidade de Estivas. Alguns equipamentos sociais (campo de futebol) do referido território e a preservação ambiental com os materiais recicláveis para a construção de objetos que possam ser utilizados como matéria prima para construção de alguns objetos do espaço de lazer.

Algumas fragilidades foram indicadas pela comunidade como a falta de incentivo financeiro por parte dos órgãos públicos. Ausência de um terreno público (área verde) destinada a construção do espaço de lazer. Para além disso, o campo de futebol foi construído em um terreno de um morador da comunidade, ou seja, não é público. Vale ressaltar que foi identificado na pesquisa, que há um interesse por parte da população, porém, não há uma mobilização da comunidade para construção do espaço de lazer.

Por meio da roda de conversa foi possível debater com a comunidade sobre as necessidades, expectativas, potencialidades e fragilidades quanto a construção de um espaço de lazer, e posteriormente foi elaborado propostas para a construção conjunta do plano de ação de construção e desenvolvimento do espaço que contribuirá para promoção da saúde dos moradores de forma concreta e sustentável. Sugere-se mais estudos nessa área e diálogos com os comunitários para potencializar a compreensão dos mesmos, ao direito e acesso a esses espaços destinados ao lazer em comunidade quilombola. 


\section{Referências}

Bacheladenski, M. S. \& Matiello Júnior, E. (2010). Contribuições do campo crítico do lazer para a promoção da saúde. Ciência \& Saúde Coletiva, Santa Cruz, $15,2569-2579$.

Batista, J. C., Ribeiro, O. C. F. \& Junior, P. C. N. (2012). Lazer e promoção de saúde: uma aproximação conveniente. LICERE-Revista do Programa de Pósgraduação Interdisciplinar em Estudos do Lazer, Minas Gerais, 15

Bardin, L. (2011). Análise de conteúdo. Edições 70, 229 p.

Brasil. Ministério da Saúde. (2006). Secretaria de Vigilância em Saúde. Política nacional de promoção da saúde. http://bvsms.saude.gov.br/bvs/publicacoes/politica_promocao_saude.pdf

Bezerra, V. M et al. (2013). Comunidades quilombolas de Vitória da Conquista, Bahia, Brasil: hipertensión y factores asociados. Cadernos de Saúde Pública, Bahia, 29, 1889-1902.

Cohen, S. C et al. (2007). Habitação saudável e ambientes favoráveis à saúde como estratégia de promoção da saúde. Ciência \& Saúde Coletiva, 12 , $191-198$.

Cruz, P. \& Araújo, R. S. (2017). Grupo de encontro comunitário como estratégia de Promoção da Saúde e construção compartilhada do saber: uma experiência de Extensão à luz da Educação Popular. Interagir: pensando a extensão24, 12-27.

Silva, R. D et al. (2012). Mais que educar...ações promotoras de saúde e ambientes saudáveis na percepção do professor da escola pública. Revista Brasileira em Promoção da Saúde, 24, 63-72.

Freitas, D. B., Miranda. S. J. \& Galvão, E. F. C. (2009). A relação do lazer com a saúde nas comunidades quilombolas de Santarém. Revista Brasileira de Ciências do Esporte, 30

Dias, C. \& Sousa, P. C. (2011). O olhar geográfico no processo de saúde e doença na zona rural de Pires do Rio-GO: usando as técnicas do geoprocessamento. Goiás.

Santos Lacerda, R. \& Mendes, G. (2018). Territorialidades, saúde e ambiente: conexões, saberes e práticas quilombolas em Sergipe, Brasil. Sustainability in Debate/Sustentabilidade em Debate, 9

Durand, M. K et al. (2016). Promoção da saúde das mulheres quilombolas: a relação com os determinantes sociais.

Ferreira, H. S et al. (2011). Nutrição e saúde das crianças das comunidades remanescentes dos quilombos no Estado de Alagoas, Brasil. Rev Panam Salud Publica, Washington, 30, http://www.scielosp.org/scielo.php?script=sci_arttext\&pid=S10204 9892011000700008\&lng=en\&nrm=iso

Gerhardt, T. E et al. (2005). A construção e a utilização do diário de campo em pesquisas científicas. International Journal of Qualitative Methods. GERAIS, Das Disposições. (1990). Lei nº 8.080, de 19 de Setembro de 1990.

Malta, D. C et al. (2014). A implementação das prioridades da Política Nacional de Promoção da Saúde, um balanço, 2006 a 2014. Ciência \& Saúde Coletiva, $19,4301-4312$.

Marquese, R. B. (2006). A dinâmica da escravidão no Brasil: resistência, tráfico negreiro e alforrias, séculos XVII a XIX. Novos estudos CEBRAP, 74, 107123.

Marinho, I., Anunciação Santos, R. \& Menezes, J. (2017). População quilombola às margens do rio são francisco: relação dos autóctones com o ambiente natural educação ambiental para a vida. RDE-Revista de Desenvolvimento Econômico.

Organização Mundial da Saúde- OMS. Carta da Organização Mundial de Saúde, 1946. http:// www.onuportugal.pt/oms.doc

Prado, R. A et al. (2013). O quotidiano e o imaginário no processo saúde-doença para as famílias quilombolas. Saúde \& Transformação Social, 4, 47-53.

Rangel, R., Miranda, A. C. M. \& Lara, L. M. (2014). Política pública de esporte e lazer no feixo: experiências de pesquisa em uma comunidade quilombola no Paraná. Licere (Online), 17

Rangel, T. L. V. (2016). Racismo ambiental às comunidades quilombolas. Revista Interdisciplinar de Direitos Humanos, 4, $129-141$.

Ribeiro, W. A. (2007). A ausência de doença e o conceito de saúde entre os gregos antigos. Saúde do Homem e da Cidade na Antiguidade Greco-Romana, Belo Horizonte e Brasília, UFMG e UNB.

Silveira, D. T. \& Córdova, F. P. (2009). Métodos de pesquisa - Planejamento e Gestão para o Desenvolvimento Rural da SEAD/UFRGS. Editora da UFRGS, p. 31-32.

Souza, M. M et al. (2015). Prevalência e fatores associados à inatividade física geral e no lazer em idosos residentes em áreas rurais. Arquivos de Ciências do Esporte, 1

Scliar, M. (2007). História do conceito de saúde. Physis: Revista de saúde coletiva, 17, 29-41.

Viana, R. N. A et al. (2017). Práticas corporais nas comunidades quilombolas: elementos para construção de políticas públicas de esportes e lazer em terras do Maranhão.

Warschauer, M. \& Carvalho, Y. M. (2014). O conceito "Intersetorialidade": contribuições ao debate a partir do Programa Lazer e Saúde da Prefeitura de Santo André/SP. Saúde e Sociedade, 23, 191-203. 
Research, Society and Development, v. 10, n. 8, e32810817221, 2021

(CC BY 4.0) | ISSN 2525-3409 | DOI: http://dx.doi.org/10.33448/rsd-v10i8.17221

World Health Organization et al. (1986). Ottawa charter for health promotion. Health promotion, International Conference on Health Promotion: the Move Towards a New Public Health.Canada, v. 1, p. iii-v.

Zank, S., Avila, J. V. C. \& Hanazaki, N. (2016). Compreendendo a relação entre saúde do ambiente e saúde humana em comunidades quilombolas de Santa Catarina. Revista brasileira de plantas medicinais, 18, 157-167. 